\title{
OBSERVATIONS ON AMEBA FEEDING ON ROTIFERS, NEMATODES AND CILIATES, AND THEIR BEAR- ING ON THE SURFACE-TENSION THEORY
}

\author{
S. O. MAST AND F. M. ROOT
}

From the Zoölogical Laboratory of the Johns Hopkins University

FIVE FIGURES

For several years past we have occasionally collected amebae in a brick-yard pond in the vicinity of Baltimore. These amebae are large lobose forms which correspond, at least superficially, with the ordinary descriptions of Ameba proteus (fig. 1). They feed almost exclusively on living organisms and thrive remarkably well in hay-infusions. When the infusoria in such cultures begin to decline the amebae begin to increase rapidly and frequently become so numerous that the substratum on which they are found appears distinctly grayish; but as the infusoria diminish the amebae decrease in numbers and eventually apparently disappear entirely, but they usually appear again if more hay and water is added so as to induce the infusoria to develop. We have found this to occur in cultures which had been inactive as long as four months. By occasionally adding a little hay and water, we have succeeded in keeping, in ordinary glass dishes, cultures of these amebae for several years. They could, no doubt, be kept indefinitely.

In connection with other work on these amebae we have incidentally observed some remarkable phenomena associated with feeding. Some of these phenomena were of such a nature that it is impossible to account for them on the assumption that movement in ameba is exclusively due to changes in surface tension as is maintained by Bütschli ('92), Ryder ('94), Jensen ('05), Verworn ('09) and others, and they seem to indicate that the rôle played by surface tension is far less significant than has 


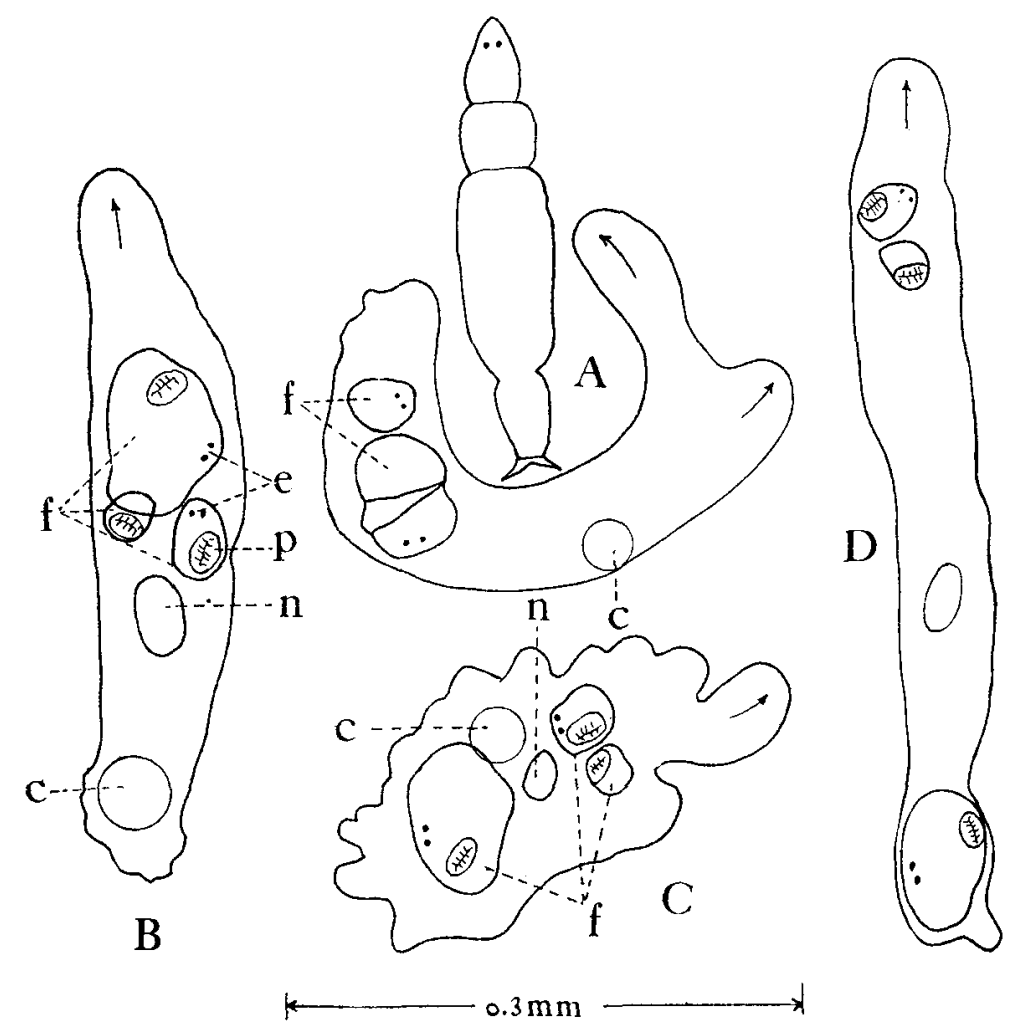

Fig. 1 Camera sketches of a rotifer and an ameba which has been feeding on rotifiers: $n$, nucleus; $c$, contractile vacuole; $f$, food vacuole, containing rotifiers; $e$, eyes; $p$, pharynx; $m m$, projected scale.

A Ameba and rotifier showing their relative size under normal conditions. Drawn November $20,12 \mathrm{~m}$. Note the large food vacuole in the ameba. It contains one large and two small rotifers, all partially digested and apparently much decreased in size. Only one of the small rotifers is shown in the sketch. Both were attached to the large one when it was captured and they were swallowed with it.

B The same ameba showing the three rotifers in the food vacuoles. Drawn November $20,12.40$ p.m.

C and D Same ameba drawn November 3, 11 and 11.05 a.m., respectively. Note that the rotifers in the food vacuoles have apparently become somewhat smaller during the preceding three days. They also became more translucent, but they were apparently still far from being completely digested. Moreover, when the ameba was discovered they were already much smaller than normal, indicating that they had been captured a considerable time earlier (compare with fig. $2 \mathrm{e}$ ). Thus it is evident that in this case it would have required much more than three days to digest the rotifers. When the last sketch was made the ameba was active and apparently in excellent condition, but three hours later it was found dead and partially disintegrated. 
been maintained by Rhumbler ('05 and '10), McClendon ('12) and others.

Feeding on rotifers. Rotifers belonging to the genus Rotifer were frequently seen in our cultures, sometimes in abundance. In one of the cultures, for more than two weeks, the amebae were found to feed almost exclusively on these animals. Fully half of the specimens examined were either attached to rotifers or contained different parts of them in their food-vacuoles (fig. 1). It seems extraordinary that an ameba should be able to capture and ingest animals relatively so powerful and active as are these rotifers.

The whole process of feeding.was not followed through in any given individual, but practically all stages in it were repeatedly observed in different ones. The following account is based upon these observations.

The rotifers in the cultures frequently became attached by means of an adhesive secretion at the posterior end and remained for considerable periods of time. While thus attached the amebae in wandering aimlessly about came in contact with them from time to time. When this occurred they usually surrounded the foot, and to this and the substratum they adhered so firmly that the rotifers could not escape. Soon after an ameba has thus surrounded the foot of a rotifer it begins to flow up around its body. This appears to cause the rotifer to contract sharply forcing the ameba back. After a time the rotifer again stretches out and then the ameba again starts to flow up around it, after which the rotifer again contracts and forces the ameba back. Thus they continue to struggle, sometimes for days (fig. 2). In the meantime the foot of the rotifer begins to digest or liquefy, and this process gradually extends out farther and farther until the animal is killed or until the injury is so great that the contractions and extensions are not strong enough to prevent the ameba from surrounding it. In several cases the rotifers were seen to move considerably after they had been swallowed. This is especially true of young individuals attached to the mother and swallowed with her. In a few cases such individuals were apparently uninjured, for they were seen to swim away after having been released by destroying the amebae (fig. 2). 
The process of digestion continues very slowly. Isolated amebae have been seen to retain vacuoles containing rotifers for three days and then the process was not complete. On Novem-

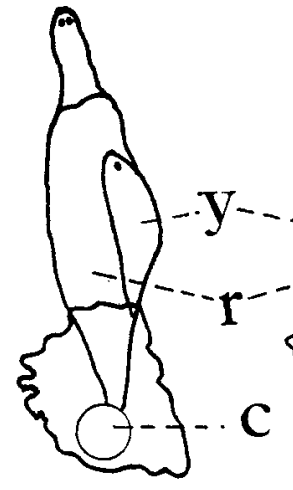

A
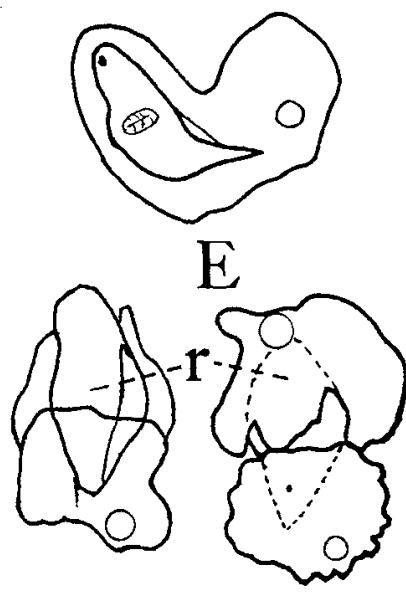

C

D

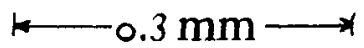

Fig. 2 Camera sketches showing an ameba feeding on a rotifer: $r$, rotifier; $y$, young rotifer attached to the mother; $c$, contractile vacuole; $m m$, projected scale.

A Ameba and rotifer as they appeared when discovered. They had evidently been united for a considerable time for the foot of the rotifer was partially digested. November 30,2 p.m.

B Same ameba and rotifer, December 1, 9 a.m. The old rotifer was dead but the young one was still alive.

$\mathrm{C}$ and $\mathrm{D}$ Same rotifer and ameba, also a second ameba, December 1, 12.28 and 1.08 p.m. respectively. At 12.28 the second ameba was rapidly flowing around the rotifier from below; at 1.08 the rotifer was nearly covered, the original. ameba being attached to the posterior and the other to the anterior end. At 2.55 p.m. the second ameba had left the rotifier and the other one was rapidly flowing away. Shortly after this the young rotifier broke through the cuticula of the mother and escaped apparently uninjured. The mother was dead, the cuticula was badly crumpled, and the foot was nearly gone. Note that the rotifer has decreased much in size.

$\mathrm{E}$ An ameba containing a rotifer which had just been swallowed. The rotifer was still alive when the sketch was made, although the foot was much injured. The ameba covered only about half of the rotifer when it was found. A little later the pseudopods began to extend rapidly and in a very short time the rotifer was entirely surrounded. 
ber $20,12 \mathrm{~m}$. a specimen containing a large rotifer, already partially digested, was isolated. Three days later the rotifer, although somewhat smaller, was still intact and apparently far from completely digested (fig. 2). During all this time the ameba was kept at room temperature, about $22^{\circ}$, and it was active and in excellent condition. Another specimen containing a rotifer was isolated December 3. Two days later this specimen had divided, but one of the daughters still retained the vacuole containing the rotifer. Similar results were obtained in a number of other cases. Greenwood ('87, p. 284) maintains that rotifers are retained one to three days.

Feeding on nematodes. Small nematodes were found in our cultures at different times but they were never very abundant. These creatures are extremely active. They almost incessantly wriggle about so violently that it seems impossible for an ameba to capture them.

The process of feeding on these creatures was observed in only one case and in this the nematode was more than half swallowed when it was discovered (fig. 3 ). The anterior end protruded and this was waving about in the most violent fashion. The process of swallowing continued, however, and fifteen minutes later the worm was entirely engulfed. The ameba then became very active, although there was no locomotion; it merely alternately elongated and contracted, extending in nearly opposite directions each succeeding time; and each time it stretched out, the nematode which constantly remained at the posterior end, was folded more closely on itself and forced into a smaller space. Thus the ameba continued to elongate first in one direction then in another until the worm was well coiled up so as to form a rather concentrated vacuole after which it slowly moved away (fig. 3). The ameba was isolated shortly after this time. Two days later it had divided and the vacuole had disappeared. It would consequently appear to require less time to digest these animals than it requires to digest rotifers.

Feeding on ciliates. The following observations were made by Mr. F. M. Root and the description is essentially as presented by him. 
When the amebae are feeding on infusoria they assume somewhat the form of a mushroom, consisting of a flat disk-like portion with a serrate edge composed of numerous short pseudopods, supported by a basal portion (fig. 4). When superficially observed the animals, in this form, appear to be perfectly quiet.

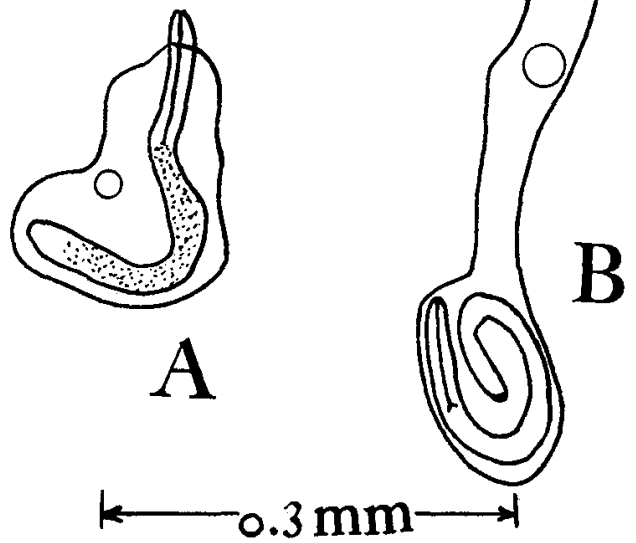

Fig. 3 Camera sketches of an ameba feeding on a nematode: $m m$, projected scale.

A Ameba and nematode as they appeared shortly after they were discovered, December 3, 10.20 a.m. At this time the protruding anterior end of the worm was violently bending back and forth. Fifteen minutes later it was entirely surrounded by the ameba.

B The same specimen, 1 p.m., showing the worm coiled up.

The pseudopods are, however, continuously in a state of flux, alternately contracting and expanding slightly.

In a given culture containing a considerable number of amebae in this form and numerous infusoria I of ten saw some of the infusoria come to rest and remain quiet for some time in the angle formed between the substratum and the projecting edge of the disk-like portion of the amebae. When this occurred a stout pseudopod usually appeared on either side of the infusorian, also 
a thin sheet of protoplasm connecting them above. These continued to flow out over the infusorian, and if it remained quiet long enough it was entirely surrounded and caught. Thus I repeatedly saw amebae capture paramecia, conjugating pairs as well as single individuals, and also some specimens of Stylonychia and Chilomonas.

There is nothing essentially new in the observations described. They are in full accord with those of Blochmann ('94, p. 87); but in connection with them I saw, on two successive days, an occurrence which I have never seen described anywhere, and which I would not have believed possible if I had not seen it. A description of the first case observed follows.

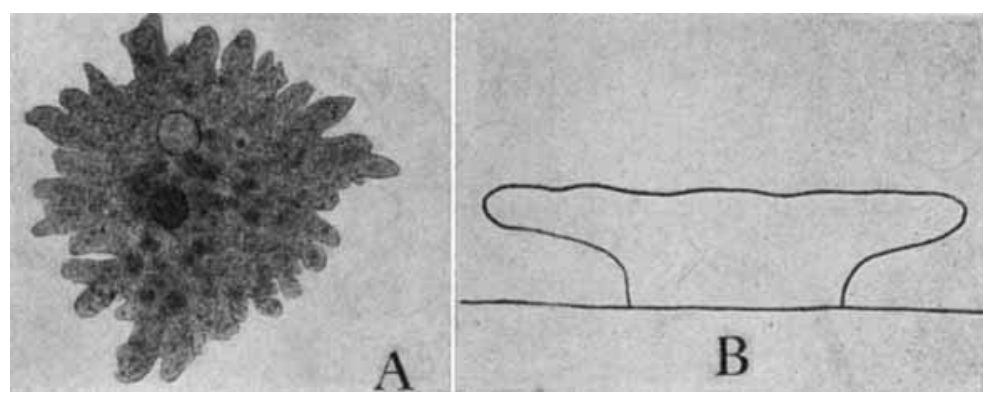

Fig. 4 Photograph and free-hand sketch of an ameba by the junior author, illustrating the form assumed in capturing infusoria.

A Surface view, B Side view.

An ameba, having the form described above, was observed in a watch-glass. A paramecium swam up to it and came to rest with the anterior end in contact with its basal portion. A pseudopod began to project on either side almost immediately as usual (fig. 5a). But when they had reached a point about midway between the two ends of the paramecium, both changed their direction of movement, and flowed directly toward the body of the paramecium, soon touching and then compressing it (fig. 5b). This compression continued until the paramecium was cut in two, one part being taken in by the ameba and the other left outside (fig. 5c). The whole process required only about ten seconds. 
During the following hour this same phenomenon was seen to occur in three other amebae; two of the infusoria captured were paramecia and the other was a stylonychia. All of these were observed in the same watch-glass. At this time I hurriedly examined the entire bottom with a binocular and found over twenty half paramecia. Since there were no other organisms present that feed on paramecia, it seems evident that all of those referred to above had been cut in two by amebae. The following

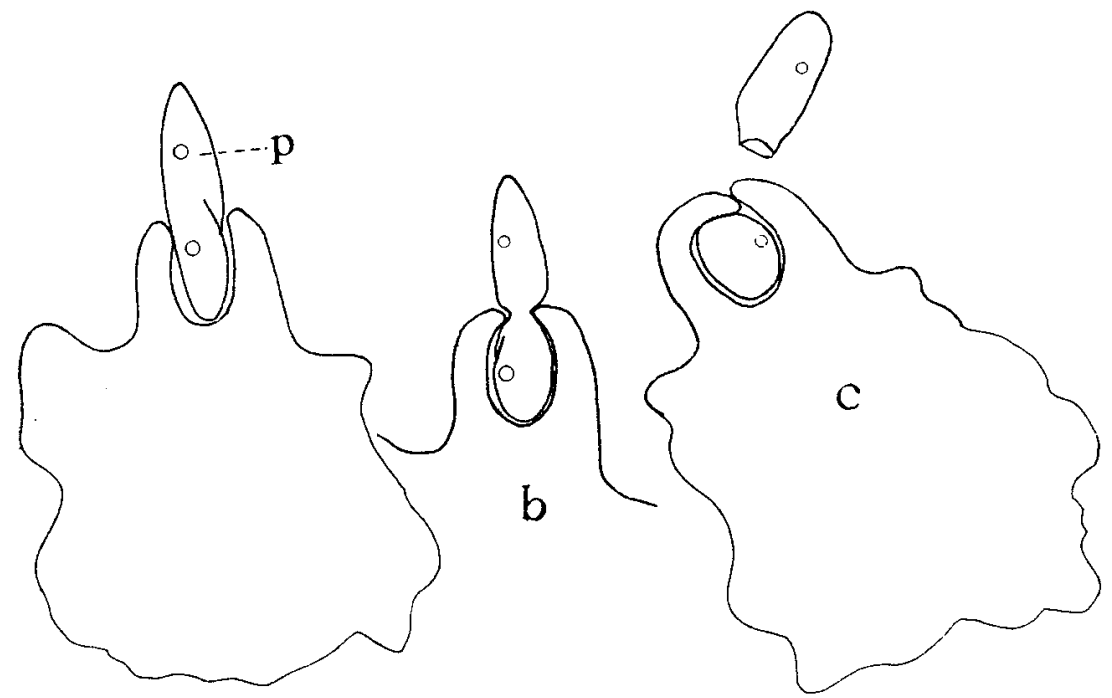

Fig. 5 Free-hand sketches made by the iunior author, showing an ameba capturing and cutting a paramecium in two; $p$, paramecium; $a, b, c$, ameba.

day this phenomenon was seen in two more cases, the prey being paramecia in both.

A year after these observations were made, one of our students, Mr. H. S. Willis, in connection with a member of the course in biology, made similar observations, but only in a single case. The ciliate captured in this case was not identified, but judging from the sketches and the description made at the time of observation, it probably was one of the hypotricha. The specimen was killed for preservation before the process was complete, the victim being not quite cut in two. In this condition it was seen 
by a considerable number of the members of our laboratory. It was, however, later unfortunately lost in the process of staining.

What bearing have these observations on the surface-tension theory? They have a bearing on this theory only in so far as it demands acceptance of the idea that the reactions of Ameba are the result of changes in surface tension.

We have no direct measurements of the surface tension of protoplasm, but on the basis of various indirect measurements and calculations, Czapek ('11, p. 41-43) concludes that it is approximately two-thirds that of water. This conclusion is supported by the facts that protoplasm is rich in organic substances, and that such substances in solution generally tend to lower the surface tension of water. Thus we would expect the surface tension of protoplasm to be considerably lower than that of water.

If this is true, it does not seem possible, a priori, that sufficient power could be developed by changes in the surface tension of a mass of protoplasm like an ameba, either to hold and engulf a struggling nematode or to master a powerful rotifer or to cleave a paramecium. In reference to the last case mentioned we are fortunate in having a method by means of which the question can be put to an experimental test.

The power required to cleave a paramecium can be ascertained with a fair degree of accuracy and from this it is possible to obtain a conception of the magnitude of the surface tension required in an ameba to perform the operation. This was accomplished as follows: One end of a glass rod was drawn out so as to produce a fine flexible fiber about $2 \mathrm{~cm}$. long. A drop of culture fluid containing numerous paramecia was mounted under a binocular. The distal end of the glass rod was now pressed down through the drop. This was repeated more or less at random until a paramecium was caught crosswise near its middle under the end of it. Pressure was then applied steadily until the paramecium was cut in two or the rod bent through an angle of more than 45 degrees.

In this way coarser and finer rods were tested until one was obtained that.would cut a majority of the paramecia when bent through 45 degrees. The pressure exerted by this rod was then 
measured by applying the end of the rod to the edge of a scale pan and ascertaining the weight necessary to balance the rod when bent through approximately 45 degrees. The results obtained with two of the rods tested are briefly summarized in table I.

TABLE I

\begin{tabular}{|c|c|c|c|c|c|c|c|c|c|c|c|c|c|c|}
\hline \multirow{3}{*}{$\begin{array}{c}\text { DIAMETER } \\
\text { OF } \\
\text { ROD AT END }\end{array}$} & \multirow{3}{*}{$\begin{array}{c}\text { LENGTH OF } \\
\text { ROD }\end{array}$} & \multicolumn{6}{|c|}{ P. AURELIA } & \multicolumn{6}{|c|}{ P. CAUDATUM } & \multirow{3}{*}{$\begin{array}{c}\text { PRESBEHE } \\
\text { APPLIED }\end{array}$} \\
\hline & & \multicolumn{3}{|c|}{$\begin{array}{l}\text { Number } \\
\text { cut in two }\end{array}$} & \multicolumn{3}{|c|}{$\begin{array}{l}\text { Number } \\
\text { not eut }\end{array}$} & \multicolumn{3}{|c|}{$\begin{array}{l}\text { Number } \\
\text { cut in two }\end{array}$} & \multicolumn{3}{|c|}{$\begin{array}{l}\text { Number } \\
\text { not cut }\end{array}$} & \\
\hline & & 0 & & 焉 & 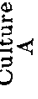 & 象 & 喿 & ${ }_{5}^{\infty}$ & m & 蛹 & 足 & 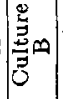 & $\begin{array}{l}\overrightarrow{8} \\
\stackrel{8}{0} \\
\dot{H}\end{array}$ & \\
\hline$m m$ & $m m$. & & & & & & & & & & & & & $m g m$. \\
\hline 0.026 & 20 & 0 & 0 & 0 & 4 & 7 & 11 & 0 & 0 & 0 & 1 & 9 & 10 & 4.5 \\
\hline 0.023 & 15 & 4 & 15 & 19 & 3 & 8 & 11 & 0 & 1 & 1 & 2 & 14 & 16 & 9 \\
\hline
\end{tabular}

These results were obtained in tests made with paramecia from two different cultures both of which contained aurelia and caudatum. They are labeled $\mathrm{A}$ and $\mathrm{B}$ respectively. By referring to the table it will be seen that the results obtained in the two sets of tests which were separated by several days are essentially the same; and it will be seen that none of the 21 paramecia caught under the weaker rod were cut in two, but that 19 of the 30 aurelia caught under the stronger rod were cut in two, while 11 were not entirely cleaved and that only one of the 17 caudatum caught under this rod was divided. In applying the pressure the hand was slowly raised and lowered so as to bring the rod to bear fully upon the paramecium. Some that could not be divided in this way were divided by drawing the rod back and forth across them but these were not recorded as cut. With the small rod, however, we were unable to divide any even in this way no matter how much the rod was bent, showing clearly that the pressure necessary to cleave a paramecium is unquestionably greater than could be applied by means of this rod, that is, greater than 4.5 mgm. The fact that 11 out of 30 could not be divided with the stronger rod without drawing the rod across them would seem to indicate that it is reasonable to accept the pressure produced by 
this rod as that necessary to cleave a majority of the animals, and to conclude that amebae in cutting paramecia in two exert a pressure as great as this.

The pressure of this rod bent through 45 degrees equaled approximately $9 \mathrm{mgm}$. as the following results show. With 9.2 mgm. in one pan the beam could not be brought to a level with the rod applied to the other; with $8.8 \mathrm{mgm}$. the beam could be brought to a level without bending the rod through quite 45 degrees; while with $9 \mathrm{mgm}$. the beam became practically level when the rod was bent through 45 degrees.

If now the cleaving of paramecium by amebae in the process of feeding is due to a change in surface tension at certain points, what must the magnitude of this change be? The answer to this question depends upon what actually occurs during the process, that is, upon whether the paramecia are cut in two by the constriction of the rim of a food-cup or by the approach of the distal ends of two pseudopods. We were unable to ascertain from our observations which of these two methods prevailed; but the bulk of the evidence at hand favors the latter. Moreover, if a cup was actually formed some factors aside from surface tension must have been involved in its formation, for it is evident that an invagination is impossible in a body the form of which is dependent solely upon surface tension. Let us however, assume a cup formed and calculate the change in surface tension necessary to account for the cutting of the paramecia on the basis of both of the methods in question.

The surface tension required in case the cutting is due to the constriction of a ring, e.g., the rim of a food-cup, can be calculated from the following formula: $P=\frac{T}{R} a b$, in which $P=$ inward pressure, $R=$ radius of ring, $a b,=$ total area in square centimeters involved in the pressure $(P)$, and $T=$ surface tension per centimeter. Now we know that to cut a paramecium it requires a pressure of approximately $9 \mathrm{mgm}$. on a glass fiber $0.023 \mathrm{~mm}$. in diameter. The length of the fiber in contact with the paramecium during the process of cutting could not exceed one-half of the circumference. It actually was continuously 
much less for as pressure was applied to the fiber the paramecium did not flatten and become wider as was expected, but the depression formed by the fiber extended down either side making it narrower at this region, so that the length of the fiber in contact was at all times less than the diameter of the paramecium and it gradually decreased as the groove made by the fiber became deeper until it was reduced nearly to zero before the paramecium was entirely divided.

Now the larger the area of the fiber in contact, the smaller will be the value of the surface tension in our calculation. We shall, however, make the present calculation on the basis of the maximum, and consider later the effect on the result of reduction due to contraction. The average diameter for 35 specimens of aurelia, the species on which the amebae fed, was found to be $0.049+\mathrm{mm}$., nearly $0.05 \mathrm{~mm}$. If we assume this to be the maximum length of fiber in contact in the process of cleaving paramecium, the area in contact will equal $(0.005 \times 0.0023)$ sq. $\mathrm{cm}$. This value may be substituted for $a b$ in the equation given above, and the pressure applied (9 mgm.) for $P$. Then $R$ in the equation will equal the radius of the paramecium being cut by the ameba. But this varies from $0.0025 \mathrm{~cm}$. to nearly zero, and the greatest pressure required does not occur until after the constriction has proceeded far enough to bring the ectosare of opposite sides in contact, that is, not until the diameter of the paramecium has been reduced to about $0.001 \mathrm{~cm}$. Assuming this statement to be valid, $R$ would equal $0.0005 \mathrm{~cm}$. Now substituting all of these values in the original equation:

$9=\frac{T(0.005 \times 0.0023)}{0.0005}, T=\frac{9 \times 0.0005}{0.005 \times 0.0023}=391 \mathrm{grams}$ per centimeter $=383.18$ dynes per centimeter or the minimum tangential tension in the rim of the food-cup required to cleave the paramecium on the basis of the first of the two methods to be considered. But in cutting the paramecium with the glass fiber the maximum pressure was applied when the length of fiber in contact was several times reduced. It is therefore evident that the tangential tension required is greater than 383.18 dynes per centimeter. But in a cup, if the constriction of the rim is 
due to surface tension, it is only the difference between the surface tension at the rim and elsewhere that can be effective. If the paramecia then were actually cut by the contraction of the rim of a food-cup due to the action of surface tension, there must have been a difference in the surface tension of the rim of the cup and that of other regions of the ameba equivalent to more than 383.18 dynes per centimeter; and to make this difference possible the surface tension of ameba must have been much larger.

If the cutting of paramecium by ameba occurred in accord with the second method mentioned above, that is, by the approach of the distal ends of two pseudopods, as the bulk of the evidence seems to indicate, and if the cutting quality of the pseudopods was the same as that of the glass fiber, then the pressure exerted by each of the pseudopods must have been approximately equal to $9 \mathrm{mgm}$. Since the pseudopods and the glass fiber were nearly all of the same size their cutting quality was probably practically the same. We may, therefore, assume that the pressure exerted by them was, if the paramecia were cut in this way, $9 \mathrm{mgm}$.

If this pressure was the result of the action of surface tension it must have been due to a reduction of the tension at the end of the pseudopods equal to $9 \mathrm{mgm}$., and the width of the surface involved must have been equal to the circumference of the pseudopods. These were approximately $0.025 \mathrm{~mm}$. in diameter having a circumference of $0.078+\mathrm{mm}$. The difference in surface tension between the end and the base of the pseudopods must therefore, if our postulates are valid, have been $9 \mathrm{mgm}$. or 8.72 dynes per $0.078+\mathrm{mm}$., which equals $1118+$ dynes per centimeter; and to produce this difference the surface tension of the ameba must have been much higher. Consequently to cut a paramecium in two in accord with this method a much higher surface tension is required than to cut it in accord with the first method.

The process in question would, therefore, require, at the very least, a surface tension considerably higher than 383 dynes per centimeter and in all probability it would require a surface tension higher than 1118 dynes per centimeter, that is, practically 
as high as that of molten platinum, the highest yet discovered. But the surface tension of protoplasm is, according to Czapek as previously stated, only approximately 50 dynes per centimeter and with a surface tension of this magnitude in ameba it is questionable whether a greater local reduction than 25 dynes per centimeter could be produced without destroying the organisms. It is therefore evident that surface tension plays a very insignificant rôle in the process of feeding described unless the protoplasm of these organisms consists of some sort of a structure that makes possible a great magnification of the effect of the surface tension. But since there is no evidence of such a structure the required power must, for the present, be sought largely in connection with other phenomena, gelation pressure, absorption pressure, adhesion, cohesion, diffusion, etc.

There is as yet little or no experimental evidence which directly bears upon the relative importance of these different factors or upon the mechanics of their regulation, although it is clear that the reactions in Ameba do not depend solely upon changes in the environment. Movement and changes in movement (responses) may undoubtedly occur without any affective external changes, such responses being entirely due to internal processes. It is also obvious that while some of the responses which are dependent upon external conditions are directly related to the environment, being local responses to local stimulation, others are not; and we are unable to conceive how some of the latter can be explained without assuming that the entire animals are involved as organized systems of considerable complexity, that there are impulses transmitted from one part of the body to another and that there is a regulatory center, in which impulses may originate and in which those originating elsewhere may be modified and controlled. This is especially true regarding much that occurs in the process of feeding on rotifers. It is also true regarding the peculiar mushroom-shape assumed when feeding on infusoria and regarding a considerable number of responses described by Jennings ('04), Kepner and Taliaferro ('13) and others. Moreover, the facts that enucleated parts of ameba do not respond at all or respond in a haphazard fashion, 
indicate, as Hofer ('90) concludes, that the nucleus acts as a regulatory center. This conclusion is also supported by the work of Mr. H. S. Willis carried on in our laboratory and now in press in the Biological Bulletin. Gruber ('12) and others, however, oppose this construction, although they obtained results similar to those obtained by Hofer and Willis.

The conclusion reached above regarding surface tension is in harmony with that reached by Jennings ('04) in his observations on the rolling movement in ameba and by Dellinger ('06) in his observations on the walking movement. It is also supported by Kepner and Taliaferro '(13) in their interesting observations on the process of feeding.

\section{SUMMARY}

1. Certain amebae at times feed almost exclusively on rotifers, at others they feed largely on paramecia.

2. They capture the rotifers by flowing around the foot at the point of attachment to the substratum. After they have surrounded the foot they begin to flow out over the body. The rotifer responds by contracting and forcing the ameba back, after which it extends again and the ameba again begins to flow out over it, etc. In the meantime the foot begins to digest and gradually the rotifer weakens. Thus they continue sometimes for days before the rotifer is swallowed.

3. When amebae are feeding on paramecia they assume a sort of mushroom shape with a serrate edge consisting of numerous short pseudopods. The paramecia tend to come to rest between and under these pseudopods by which they are usually surrounded, but sometimes the ends of the pseudopods approach each other before they are fully extended and cut the paramecium in two.

4. To cut a paramecium in two with a fine glass fiber it requires a pressure of approximately $9 \mathrm{mgm}$. If the pseudopods have the same cutting quality as the glass fiber and if their movement is due to a change of surface tension, it requires to perform the work involved, a reduction in surface tension of at least 1118 dynes per centimeter at the tips of the pseudopods. 
5. If the ends of the pseudopods fuse so as to take on the form of a ring around the paramecium and if the cutting is due to constriction in this ring, and if the constriction in this ring is due to a change in surface tension, the work involved requires a minimum reduction along the inner surface of the ring of at least 383 dynes per centimeter.

6. The bulk of evidence at hand seems to indicate that the paramecia are divided by the approach of two pseudopods. To account for the process on the basis of the surface tension theory therefore, the surface tension of the amebae would have to be, at the very least, much higher than 383 dynes per centimeter and in all probability considerably higher than 1118 dynes per centimeter. The surface tension of protoplasm is, however, only approximately 50 dynes per centimeter. It is, therefore, probably at best an insignificant factor in the process of feeding in ameba. 


\section{LITERATURE CITED}

Blochmann, F. 1894 Kleine Mitteilungen über Protozoen. Biol. Centralb. Bd. 14, S. 82-91.

BÜTSCHLI, O. 1892 Untersuchungen über mikroskopische Schäume und das Protoplasma. Leipzig, $234 \mathrm{~S}$.

Czapex, Frederick 1911 Chemical phenomena in life. London and New York, $148 \mathrm{pp}$.

Dellinger, O. P. 1906 Locomotion of amoebae and allied forms. Jour. Exp. Zoöl., vol. 3, p. 337-357.

Greenwood, M. 1887 On the digestive process in some Rhizopods. Jour. Phys., vol. 8, pp. 263-287.

Grdber, K. 1912 Biologische und Experimentelle Untersuchungen an Amoeba proteus. Arch f. Protistenkunde. Bd. 25, S. 316-376.

Hofer, Bruno 1890 Experimentelle Untersuchungen über den Einfluss des Kerns auf das Protoplasma. Jen. Zeitschr. f. Naturw., Bd. 24, S. $105-176$.

Jennings, H. S. 1904 Contributions to the study of the behavior of lower organisms. Carnegie Inst. of Washington, Pub. No. 16, 256 pp.

Jensen, P. 1905 Zur Theorie der Protoplasmabewegung und über die Auffassung des Protoplasmas als chemisches System. Anatom. Hefte (Merkel u. Bonnet), Bd. 27, Heft, 83, S. 831-858.

KePNer, Wm. A. AND Taliaferro, W. H. 1913 Reactions of Amoeba proteus to food. Biol. Bull., vol. 24, pp. 411-428.

MCClendon, J. F. 1912 The osmotic and surface tension phenomena of living elements and their physiological significance. Biol. Bull., vol. 22, pp. 113-204.

Rhumbler, L. 1905 Zur Theorie der Oberflächenkräfte der Amöben. Zeit. f. wiss. Zool., Bd. 83, S. 1-52.

1910 Die verschiedenartigen Nahrungsaufnahmen bei Amöben als Folge verschiedener Colloidalzustände ihrer Oberflächen. Arch. f. Entw.-Mech., Bd. 30, S. 194-220.

RYDeR, J. A. 1893 Dynamics in evolution. Biological Lectures, Woods Hole, pp. 63-81.

Verworn, MAX 1909 Allgemeine Physiologie. Fünfte Auflage, Jena, 742 S. 